\title{
EFEITO DAS DIFERENTES MADEIRAS NO ISOLAMENTO ACÚSTICO
}

\author{
Ançano Loschi Neto ${ }^{1}$, José Reinaldo Moreira da Silva ${ }^{2}$, José Tarcísio Lima ${ }^{2}$, \\ Giovanni Francisco Rabelo ${ }^{3}$ \\ ${ }^{1}$ Eng. Florestal, M.Sc., Lavras, MG, Brasil - aloschi@gmail.com \\ ${ }^{2}$ Eng. Florestal, Dr., Depto. de Engenharia Florestal, UFLA, Lavras, MG, Brasil - jreinaldo@ufla.br - jtlima@ufla.br \\ ${ }^{3}$ Eng. Eletricista, Dr., Depto. de Engenharia, UFLA, Lavras, MG, Brasil - giovannirabelo@yahoo.com.br
}

Recebido para publicação: 04/09/2007 - Aceito para publicação: 18/03/2008

\begin{abstract}
Resumo
$\mathrm{O}$ isolamento acústico depende das propriedades do material, principalmente dimensões, formas umidade e densidade. O objetivo deste trabalho foi avaliar o isolamento sonoro proporcionado por madeiras de diferentes densidades e verificar o comportamento do isolamento por meio da análise espectral. Foram selecionadas madeiras do gênero Eucalyptus, Dipteryx (cumaru) e Pinus, em cinco grupos de distintas densidades. Foram montadas barreiras acústicas, em forma de cubos ocos com arestas internas de $60 \mathrm{~cm}$. A avaliação consistiu em emitir uma onda gerada por um computador por meio de um alto-falante localizado dentro da barreira acústica e captá-la por um microfone localizado a um metro de distância. O nível de pressão sonora foi medido com um decibelímetro. A maior atenuação acústica $(25,7 \mathrm{~dB}(\mathrm{~A}))$ foi apresentada pela madeira de Eucalyptus grandis $\left(0,880 \mathrm{~g} / \mathrm{cm}^{3}\right)$, e o pior desempenho foi apresentado pelo Pinus sp, com $15 \mathrm{~dB}(\mathrm{~A})$. Essas madeiras apresentaram maior e menor densidade, respectivamente.
\end{abstract}

Palavras-chave: Madeira; densidade; isolamento acústico.

\begin{abstract}
Effect of different wood in the acoustic isolation. Acoustic isolation depends upon characteristics of the material, mainly on the dimensions, shape and density. The objective of this work was to evaluate the sound isolation provided by woods of different densities and verify the behavior of the isolation through spectral analysis. They were selected woods of the genera Eucalytus, Pinus and Dipteryx (Cumaru) with five groups of distinct densities. Boxes of $60 \mathrm{~cm}$ of internal edges were mounted. The equipment for the evaluation was mounted in a wide and grassy field. The experiment consisted in emitting a form of wave, generated in a computer by means of a loudspeaker localized inside the wooden box and picked it up by a microphone situated at a meter away. The level of sound pressure was measured with a decibelimeter. The wood which promoted the highest acoustic attenuation was E. grandis $(0.880 \mathrm{~g} / \mathrm{cm} 3)$ with an attenuation of $25.7 \mathrm{~dB}(\mathrm{~A})$, but the wood of worst performance was Pinus sp with $15 \mathrm{~dB}$ (A). These woods presenting the highest and poorest density respectively.

Keywords: Wood; density; acoustic isolation.
\end{abstract}

\section{INTRODUÇÃO}

O homem, ao receber informações do ambiente e assimilá-las, pode reagir de maneira favorável ou não ao estímulo. Uma das grandes causas da queda na qualidade de percepção do ambiente é a poluição. Ela possui diversas formas de manifestação. Destacam-se a poluição atmosférica, a visual, a provocada pelo lixo e a sonora.

A perda de audição induzida por ruído (PAIR) em ambientes de trabalho tem como causa a exposição a elevados níveis de pressão sonora - acima de $85 \mathrm{~dB}(\mathrm{~A})$ - por um determinado tempo. Quanto mais elevado o nível de pressão sonora, menor o tempo permitido de sua exposição, considerando-se que o indivíduo encontra-se sem equipamento de proteção individual.

Para um ambiente ser considerado ideal para a permanência de pessoas, do ponto de vista acústico, deve-se atentar para certas premissas, as quais são tratadas pela acústica arquitetônica. O grande 
foco é dado em dois sentidos: controle de sons no interior dos ambientes e isolamento contra ruídos externos.

As características sonoras estabelecidas no interior de um espaço fechado são condicionadas tanto pela concepção arquitetônica como pelos revestimentos, pelo mobiliário, pelo equipamento e por tudo o mais que compõe seu interior. Assim, a madeira, material por excelência dos revestimentos, assume papel determinante no desempenho acústico desses locais, podendo ser utilizada para promover a absorção sonora ou as reflexões difusas, condicionando o campo sonoro estabelecido no interior dos ambientes.

Materiais usados para isolamento apresentam alta densidade, dificultando a passagem da onda sonora. Sendo assim, madeiras com densidades elevadas apresentam maiores valores de isolamento quando comparadas com madeiras de densidade mais baixa. $\mathrm{O}$ objetivo deste trabalho foi avaliar o isolamento sonoro proporcionado por madeiras de diferentes densidades.

\section{REVISÃO BIBLIOGRÁFICA}

\section{Som e ruído}

O som é toda e qualquer vibração ou onda mecânica que se propaga em um meio dotado de forças internas (elástico, viscoso etc.) capaz de produzir no homem uma sensação auditiva (ABNT, 1992).

Ao encontrar uma superfície, a onda sonora sofre alterações (Figura 1), que estão relacionadas ao tipo de material constituinte dessa superfície. Essas alterações são definidas como absorção, especialmente nas superfícies mais macias e porosas (Figura 1a), transmissão de um lado para outro, por meio da ressonância (Figura 1b) e reflexão, quando muda a direção devido à convexidade ou concavidade da superfície sobre a qual incide, ou ainda quando a onda apresenta um determinado ângulo de incidência (Figura 1c).

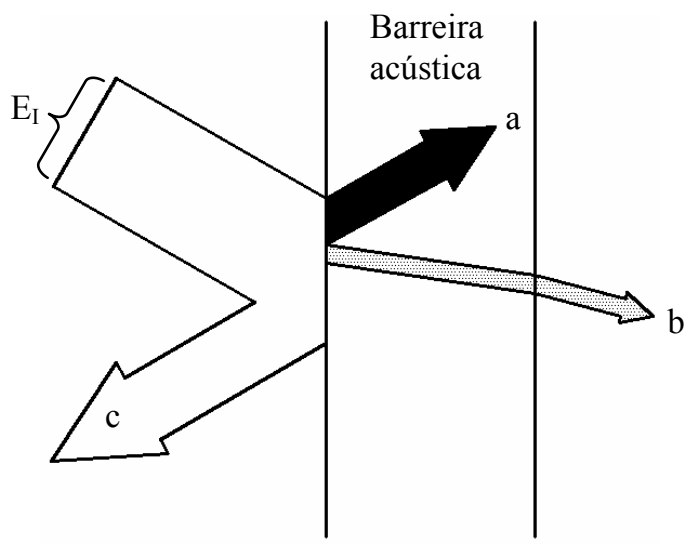

Fonte: Adaptado de Fernandes (2002)

Figura 1. Alterações sofridas por uma onda sonora ao entrar em contato com uma superfície. $\mathrm{E}_{\mathrm{I}}$ : energia incidente; a: energia absorvida; b: energia transmitida; c: energia refletida.

Figure 1. Alterations suffered for a sonorous wave when finding a surface. $\mathrm{E}_{\mathrm{I}}$ : incident energy; a: absorption; b: transmission; c: reflection.

Dentre as grandezas acústicas, as mais importantes são a intensidade, a potência e a pressão sonora. A intensidade sonora é definida como a quantidade de energia sonora que atravessa uma unidade de área durante certo tempo. Potência sonora é uma característica intrínseca da fonte sonora. Seu valor independe do meio e sua unidade é Watts. Segundo Bistafa (2006), a pressão sonora é a grandeza mais importante para caracterizar os efeitos do ruído em seres humanos. Sua unidade é o Pascal (Pa). A pressão sonora pode ser obtida de maneira simples, por aparelhos como o decibelímetro, que converte seus valores para a escala de decibéis, facilitando sua interpretação. Seus valores dependem da distância entre o ponto em que é medida e a fonte sonora, além da quantidade de energia sonora absorvida pelo meio. A determinação do nível de pressão sonora (NPS), em decibéis, encontra-se descrito pela Equação 1 (BISTAFA, 2006). 


$$
N I S=20 \cdot \log \left(\frac{I}{I_{0}}\right)
$$

Em que: $\quad$ NIS = nível de intensidade sonora.

$20=$ constante.

$\mathrm{I}=$ estímulo sonoro, em $\mathrm{W} / \mathrm{m}^{2}$.

$\mathrm{I}_{0}=$ valor mínimo do estímulo $\left(10^{-12} \mathrm{~W} / \mathrm{m}^{2}\right)$.

Ruído é qualquer som indesejável. Sua definição se baseia na condição do ouvinte, seu estado de saúde, humor, cultura etc. O ruído afeta o homem de várias formas. Ele causa prejuízos ao funcionamento do sistema auditivo e compromete a atividade física, fisiológica e mental do indivíduo a ele exposto (CLÍMACO, 2003). Oiticica; Gomes (2004) constataram que, devido à formação de ambientes insalubres em salas de aulas, os professores passam por estágios que variam desde um estresse leve até estresse degenerativo. Foi observado também um desgaste das cordas vocais dos professores por conta de alto índice de ruído nas salas de aula. A partir de $35 \mathrm{~dB}(\mathrm{~A})$, o ruído provoca reações vegetativas. Em longo prazo e em níveis acima de $70 \mathrm{~dB}(\mathrm{~A})$, o ruído pode causar hipertensão arterial, secreção elevada de hormônios corticosteróides, estresse, infarto etc. (PIMENTEL-SOUZA, 2000).

\section{Acústica de ambientes}

Para ser considerado ideal para a permanência de pessoas, um ambiente deve apresentar certos níveis de conforto. Os principais pontos tratados nessa área são o isolamento contra o ruído e o controle do som no interior do ambiente. $\mathrm{O}$ isolamento sonoro (ou atenuação sonora) consiste em impedir, ou pelo menos reduzir, a propagação de sons entre dois ambientes distintos.

De acordo com Paixão (2002), para se conseguir um isolamento adequado é necessário o uso de materiais que apresentam densidades mais elevadas, de acordo com a Lei da Massa, apresentada na Equação 2. De acordo com essa equação, quanto maior a massa do material, maior o isolamento, e quanto mais alta a freqüência, mais fácil de ser isolada. Cada vez que a massa de uma superfície é dobrada, ocorre um incremento de $4 \mathrm{a} 5 \mathrm{~dB}$ na sua capacidade atenuadora (SILVA, 2005).

$$
\mathrm{PT}=-42,4+20 \log (\mathrm{M} * \mathrm{f})
$$

Em que: $\quad \mathrm{PT}=$ perda de transmissão sonora $(\mathrm{dB})$.

$20=$ constante.

$\mathrm{M}=$ massa por unidade de área $\left(\mathrm{kg} / \mathrm{m}^{2}\right)$.

$\mathrm{f}=$ freqüência $(\mathrm{Hz})$.

Considerando-se que a característica do isolamento não é a mesma em todas as freqüências, torna-se necessário a medição da intensidade do ruído em faixas de freqüência de $1 / 3$ de oitava, abrangendo principalmente o intervalo entre as freqüências de 64 e $4.000 \mathrm{~Hz}$.

A tabela 1 apresenta o isolamento proporcionado por alguns materiais usados em construção. Nesse caso, foi tirada a média dos valores em cada banda de oitava, fornecendo assim um valor único representativo do total isolado pelo material (FERNANDES, 2002).

Tabela 1. Atenuação do som de algumas superfícies.

Table 1. Attenuation of sound of some surfaces.

\begin{tabular}{lc}
\hline Material & Atenuação, em dB(A) \\
\hline Parede de tijolo maciço com $45 \mathrm{~cm}$ de espessura & 55 \\
Porta de madeira maciça dupla com $5 \mathrm{~cm}$ por folha & 45 \\
Janela de vidros duplos de 3 mm separados $20 \mathrm{~cm}$ & 45 \\
Porta de madeira maciça de $5 \mathrm{~cm}$ de espessura & 30 \\
Porta comum sem vedação no batente & 15 \\
\hline
\end{tabular}

Fonte: Fernandes (2002)

Ao contrário do que ocorre ao ar livre, a onda sonora dentro de um ambiente sofre interferência das ondas refletidas pelas superfícies que demarcam o recinto (BISTAFA, 2006). O tratamento interno consiste em distribuir o som de forma homogênea pelo ambiente, livre de qualquer interferência ou efeito 
que cause desconforto (OITICICA; GOMES, 2004). O principal parâmetro relacionado à caracterização interna do ambiente é o tempo de reverberação (RT60). Ele é definido como o tempo gasto para a energia sonora decair $60 \mathrm{~dB}(\mathrm{~A})$, contado a partir de sua interrupção. Está relacionado com o volume físico, com os materiais constituintes das superfícies dos objetos presentes no ambiente e também com as paredes, teto e piso (CLÍMACO, 2003). Assim como para o nível de ruído, o tempo de reverberação ideal varia de acordo com o uso proposto para o ambiente. $\mathrm{Na}$ tabela 2 estão relacionados alguns tempos de reverberação considerados ideais para alguns ambientes encontrados em instalações escolares.

Tabela 2. Tempos de reverberação adequados (segundos), para alguns ambientes.

Table 2. Adequate reverberation times (seconds) for some environments.

\begin{tabular}{lc}
\hline Ambientes & Intervalo de tempo ideal de reverberação (segundos) \\
\hline Ensaios musicais & $0,6-1,2$ \\
Auditórios & $1,0-1,5$ \\
Ginásios & $1,2-1,6$ \\
Lanchonetes & $0,8-1,2$ \\
Salas de aula & $0,4-0,6$ \\
\hline Fonte: Adaptado de Seep et al. (2002)
\end{tabular}

Materiais utilizados na correção acústica interna de ambientes são fibrosos ou porosos. A absorção da energia sonora ocorre pelo atrito das moléculas de ar no interior do material na passagem da onda sonora. O coeficiente de absorção de cada material obedece à mesma relação encontrada no isolamento sonoro, em que ocorre uma variação do valor para cada freqüência. $\mathrm{O}$ ambiente de trabalho requer condições ideais para o melhor rendimento dos profissionais envolvidos. Além disso, condições adversas podem causar danos à saúde dos trabalhadores.

Segundo Bistafa (2006), a perda de audição induzida por ruído (PAIR) ocorre pela associação de dois fatores: nível de ruído e tempo de exposição a ele. Segundo a Norma Regulamentadora $\mathrm{n}^{\mathrm{o}} 15$, a exposição a níveis de ruído iguais ou acima de $85 \mathrm{~dB}(\mathrm{~A})$ já requer do trabalhador atitudes defensivas. Para esse valor, o tempo de exposição máxima permitida é de 8 horas. Acima desse período, o ambiente já é considerado insalubre. Quanto maior o nível de ruído, menor será o tempo máximo de exposição permitido. A cada aumento de $5 \mathrm{~dB}(\mathrm{~A})$, ocorre uma redução do tempo pela metade até $115 \mathrm{~dB}(\mathrm{~A})$, ponto em que o tempo de exposição é de 7 minutos. Acima desse nível não é permitida a exposição sem a devida proteção, sob risco de dano grave à audição.

\section{Propriedades fundamentais da madeira}

As propriedades fundamentais da madeira são descritas como aquelas relacionadas à sua formação. São elas que irão determinar a durabilidade, a identificação, a resistência e também a qualidade dos produtos gerados a partir de uma árvore. São agrupadas em propriedades anatômicas, físicas, mecânicas e químicas. Dentre as propriedades físicas da madeira, as mais estudadas são a umidade e as densidades básica e aparente. Segundo Stewart; Polak (1975), a densidade aparente da madeira, definida como a relação entre a massa e o volume a uma determinada condição de umidade, é uma das mais representativas propriedades físicas da madeira. A densidade básica também é considerada uma das características mais importantes, tendo como principais pontos a facilidade na determinação e sua relação com as demais propriedades da madeira. É definida como a relação entre a massa absolutamente seca e o volume em máxima expansão da madeira.

Segundo Carrasco; Azevedo Júnior (2003), a velocidade de propagação da onda ultrassônica é afetada pela densidade da madeira. Um aumento desse valor, com umidade constante ao longo da amostra, provoca um aumento na velocidade de propagação. Esse fato se deve à deposição de celulose na face interna da parede celular, que causa um aumento significativo da rigidez. Assim, a esperada atenuação causada pelo aumento da densidade é compensada por esse parâmetro.

\section{Propriedades acústicas da madeira}

De acordo com Araújo (2002), as propriedades acústicas da madeira, relacionadas aos princípios de ressonância e às propriedades de radiação do som, foram aplicadas por muito tempo, apesar de não serem cientificamente comprovadas. 
Segundo Bucur, citado por Shimoyama (2005), a avaliação das propriedades acústicas da madeira pode indicar a anisotropia desse material, considerando sua relação com as características anatômicas.

Para a confecção de instrumentos musicais, os valores de resistência à propagação da onda sonora e de resistência à irradiação sonora devem ser cuidadosamente estudados (SOUZA, 1983). Esses valores estão intimamente ligados ao módulo de elasticidade dinâmico da madeira e servem de embasamento para uma série de experimentos relacionados à determinação de propriedades da madeira por meio de métodos acústicos, tais como ultrassom e propagação de ondas de tensão.

A maioria dos ensaios acústicos requer a utilização de ruídos padronizados, os quais apresentam características peculiares. Os mais utilizados são o ruído branco e o ruído rosa. A principal diferença entre esses dois tipos de ruído está relacionada à densidade de energia sonora presente ao longo do espectro de freqüência. Enquanto o ruído branco apresenta uma quantidade de energia constante para todas as freqüências, o ruído rosa apresenta uma queda de $3 \mathrm{~dB}$ por oitava (FERNANDES, 2002).

\section{MATERIAL E MÉTODOS}

Foram usadas madeiras em cinco distintos grupos de densidades, dos gêneros Dipteryx (cumaru), Eucalyptus e Pinus. Para o Eucalyptus, foram utilizados três clones do teste de procedências (PRODEPEF), implantado em 1975, na UFLA. As toras de Eucalyptus foram desdobradas, obtendo-se tábuas de $300 \times 2,5 \mathrm{~cm}(\mathrm{C} \times \mathrm{E})$ e largura variável. A seguir, procedeu-se à secagem, em estufa, até 14\% de umidade. Para Dipteryx (cumaru) e Pinus, foram adquiridas tábuas de 400 x 30 × 2,5 cm (C x L x E), numa madeireira de Lavras/MG. Todo material foi processado no Laboratório de Usinagem da Madeira (DCF/UFLA), obtendo-se tábuas de $74 \times 15 \times 1,7 \mathrm{~cm}$, as quais foram entabicadas num galpão coberto até atingirem a umidade de equilíbrio de, aproximadamente, $15 \%$. O acompanhamento foi pela perda de massa. Posteriormente, foi medida a velocidade de propagação das ondas de tensão longitudinalmente à grã da madeira, utilizando-se o stress wave timer (Figura 2), em três pontos eqüidistantes ao longo da largura das peças, em três repetições.

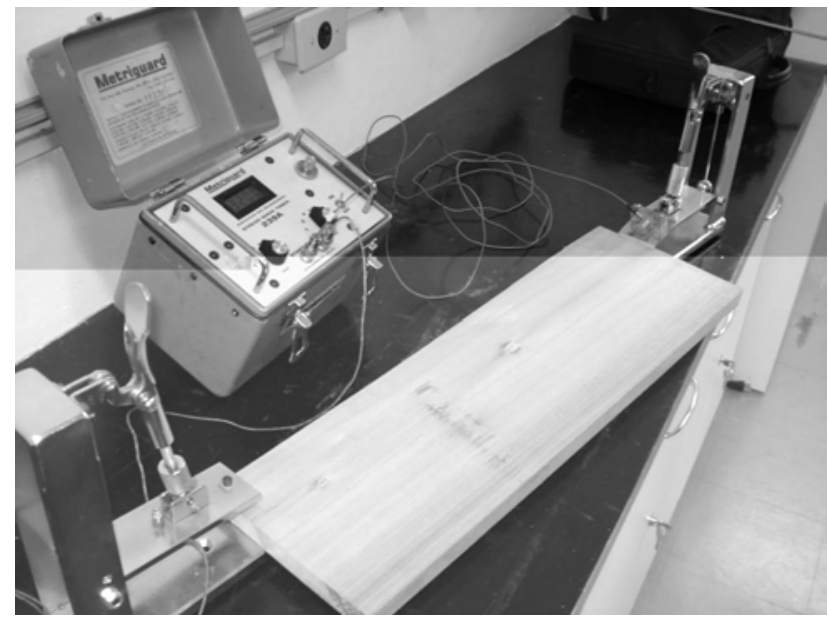

Figura 2. Determinação da velocidade de propagação das ondas na madeira.

Figure 2. Determination of the propagation velocity of the waves in the wood.

No Laboratório de Usinagem da Madeira (DCF/UFLA), foram confeccionadas as barreiras acústicas. Para tanto, produziram-se painéis com emendas laterais, tipo "macho-fêmea", para se evitar perda de energia sonora. As barreiras acústicas produzidas apresentaram a forma de cubos ocos com arestas internas de $60 \mathrm{~cm}$, com tampa móvel, afixada por 4 parafusos em cada lado. Concomitantemente, amostras de cada tábua (resíduos dos cortes) foram retiradas e armazenadas em sacos plásticos. Essas amostras serviram para determinação das densidades aparentes e das umidades, conforme NBR 11941 (2003) e NBR 7190 (1997), respectivamente. 
Para avaliação do isolamento acústico, foram utilizados um microcomputador, um amplificador/receiver, um alto falante (50 W RMS e resposta de freqüência de 22 a $30.000 \mathrm{~Hz}$ ), um microfone de cápsula dinâmica (resposta de freqüência de 40 a $18.000 \mathrm{~Hz}$ ) e um decibelímetro, conforme recomendações da IEC 651. Na saída de áudio do microcomputador foi acoplado o alto-falante ligado ao amplificador/receiver, e na entrada da placa de som, o microfone. Desse modo, todo som transmitido pelo computador era também gravado por ele. O alto-falante foi fixado no interior da barreira acústica por meio de um pedestal para microfone. $\mathrm{O}$ experimento foi conduzido em terreno gramado e plano, de modo a se evitar a reflexão dos sons que incidem no piso (HERRERA et al., 2002). Por meio de um aplicativo computacional, foi gerado um arquivo de áudio contendo um ruído aleatório. A pressão sonora foi medida com o decibelímetro a $100 \mathrm{~cm}$ de distância do alto-falante. Foram realizadas 90 leituras para o cálculo do nível de pressão sonora equivalente $\left(\mathrm{L}_{\mathrm{eq}}\right)$. Todas as medições foram executadas na ausência de vento e de precipitação.

O alto-falante foi colocado dentro da barreira acústica (Figura 3) e o procedimento anterior foi repetido, mantendo-se constante a intensidade do som e as distâncias. Foram feitas 90 leituras para cada barreira acústica das diferentes madeiras. Com o decibelímetro, mediu-se o ruído de fundo, seguindo as recomendações da Norma Técnica L11.031 (1986) da Companhia de Tecnologia de Saneamento Ambiental (CETESB). Os dados foram analisados estatisticamente pelo teste de Student-Newman-Kuels (SNK), a 5\% de significância.

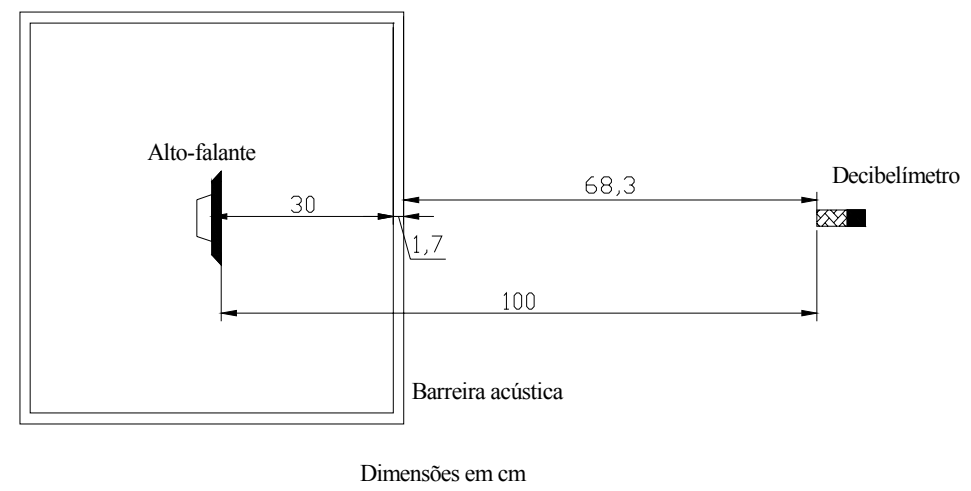

Figura 3. Desenho esquemático da visão superior das leituras do isolamento acústico. Figure 3. Schematic drawing in upper view of the acoustic isolation measurement.

\section{RESULTADOS E DISCUSSÃO}

Os valores médios de umidade e densidades aparente e básica das madeiras usadas na confecção das barreiras acústicas são apresentadas na tabela 3. Observa-se que a umidade apresentou-se uniforme, fato que mostra a não-interferência dessa variável no isolamento acústico, conforme alertado por Cruz (2006) e Carrasco; Azevedo Júnior (2003). Os valores da densidade aparente obtidos apresentaram uma adequada distribuição, possibilitando a avaliação satisfatória em cinco classes de madeiras com diferentes densidades.

Na tabela 4 encontra-se a análise de variância para a velocidade de propagação das ondas de tensão. Verifica-se que houve diferença significativa para as diferentes madeiras. Esse fato já era esperado, devido à variação da densidade nas diferentes madeiras utilizadas na confecção das barreiras acústicas. Os valores mínimos e máximos e a comparação múltipla das médias encontram-se na tabela 5. Observa-se uma pequena variação entre os três pontos de leitura na mesma tábua, fato que, conforme Cruz (2006) e Carrasco; Azevedo Júnior (2003), pode estar associado à pequena variação de umidade e de densidade. Para a madeira de E. grandis de $0,880 \mathrm{~g} / \mathrm{cm}^{3}$, que apresentou o menor percentual de variação, o maior e o menor valor foram de 4242 e $4217 \mathrm{~m} / \mathrm{s}$, respectivamente. Observa-se que há um incremento na velocidade de propagação com o aumento da densidade. Segundo Carrasco; Azevedo Júnior (2003), isso ocorre pelo fato de o aumento da densidade acontecer devido a um aumento da deposição de celulose na parede celular. O resultado é um acréscimo nos valores de rigidez do material, com conseqüente aumento da velocidade de propagação das ondas de tensão. 
Tabela 3. Valores médios de umidade e de densidades aparente e básica das madeiras utilizadas na confecção das barreiras acústicas.

Table 3. Mean values of moistures, apparent and basic densities of the woods used for construction acoustics obstacles.

\begin{tabular}{lccc}
\hline \multirow{2}{*}{ Espécie } & \multirow{2}{*}{ Umidade (\%) } & \multicolumn{2}{c}{ Densidade $\mathbf{( g / \mathbf { c m } ^ { \mathbf { 3 } } )}$} \\
\cline { 3 - 4 } & & Aparente & Básica \\
\hline E. grandis -1 & 14 & 0,728 & 0,596 \\
E. grandis -2 & 14 & 0,799 & 0,612 \\
E. grandis -3 & 15 & 0,880 & 0,609 \\
Dipteryx sp. (cumaru) & 14 & 0,817 & -- \\
Pinus sp. & 14 & 0,445 & -- \\
\hline
\end{tabular}

Tabela 4. Análise de variância para a velocidade de propagação de ondas de tensão.

Table 4. Variance analysis for velocity of propagation of the stress wave.

\begin{tabular}{lccc}
\hline Fonte de variação & Grau de liberdade & Quadrado Médio & Fc \\
\hline Espécie & 4 & 415853,94 & $248,205^{*}$ \\
Erro & 10 & 1675,44 & \\
\hline Total & 14 & & \\
\hline
\end{tabular}

Tabela 5. Velocidades mínimas e máximas de propagação de ondas de tensão, para as madeiras utilizadas na confecção das barreiras acústicas.

Table 5. Minimum and maximum values of the velocity of propagation of the stress wave, for wood used in the confection of the acoustics obstacle.

\begin{tabular}{lccc}
\hline \multirow{2}{*}{ Madeira } & \multicolumn{3}{c}{ Velocidade de propagação das ondas de tensão (m/s) } \\
\cline { 2 - 4 } & Mínima & Máxima & Médias \\
\hline Pinus & 3595 & 3689 & $3182 \mathrm{a}$ \\
Dipteryx $($ cumaru) & 3729 & 3775 & $3630 \mathrm{~b}$ \\
E. grandis $\left(0,728 \mathrm{~g} / \mathrm{cm}^{3}\right)$ & 3129 & 3239 & $3684 \mathrm{bc}$ \\
E. grandis $\left(0,799 \mathrm{~g} / \mathrm{cm}^{3}\right)$ & 3652 & 3735 & $3753 \mathrm{c}$ \\
E. grandis $\left(0,880 \mathrm{~g} / \mathrm{cm}^{3}\right)$ & 4217 & 4242 & $4228 \mathrm{~d}$ \\
\hline
\end{tabular}

Médias seguidas de, pelo menos, uma mesma letra não diferem entre si, a 5\% de significância, pelo teste de Tukey.

Os valores mínimos, médios e máximos de pressão sonora são apresentados na tabela 6 . Observa-se uma relação média de 40,9 dB(A) entre o sinal do alto-falante emitido sem a barreira acústica e o ruído de fundo. Esse aspecto proporciona uma boa amplitude para a avaliação dos ruídos intermediários, posto que, segundo Seep et al. (2002), com uma diferença acima de $10 \mathrm{~dB}(\mathrm{~A})$ evita-se que o som do alto-falante seja mascarado pelos ruídos de fundo.

Tabela 6. Valores mínimos, médios e máximos dos níveis de pressão sonora obtidos com o decibelímetro, em $\mathrm{dB}(\mathrm{A})$.

Table 6. Minimum, mean and maximum values of the levels of sound pressure obtained through the decibelmeter in $\mathrm{dB}(\mathrm{A})$.

\begin{tabular}{lccc}
\hline \multirow{2}{*}{ Tratamento } & \multicolumn{3}{c}{ Pressão sonora dB(A) } \\
\cline { 2 - 4 } & Mínimo & Médio & Máximo \\
\hline E. grandis $\left(0,728 \mathrm{~g} / \mathrm{cm}^{3}\right)$ & 64,2 & 65,4 & 66,8 \\
E. grandis $\left(0,799 \mathrm{~g} / \mathrm{cm}^{3}\right)$ & 61,0 & 62,2 & 63,4 \\
E. grandis $\left(0,880 \mathrm{~g} / \mathrm{cm}^{3}\right)$ & 54,5 & 57,8 & 60,4 \\
Dipteryx sp. (cumaru) & 63,5 & 64,9 & 66,1 \\
Pinus sp. & 66,6 & 68,5 & 69,7 \\
Ruído de fundo & 40,1 & 43,4 & 46,1 \\
Sem barreira acústica & 81,1 & 83,5 & 86,4 \\
\hline
\end{tabular}


De acordo com figura 4, observa-se uma pequena variação entre os valores máximos e mínimos em cada tratamento. Porém, apesar de numericamente muito próximos, esses valores apresentam uma diferença significativa do ponto de vista acústico. De acordo com a escala utilizada, a cada 3 decibéis tem-se o dobro de energia sonora, e 1 decibel é a mínima variação perceptível pelo sistema auditivo humano.

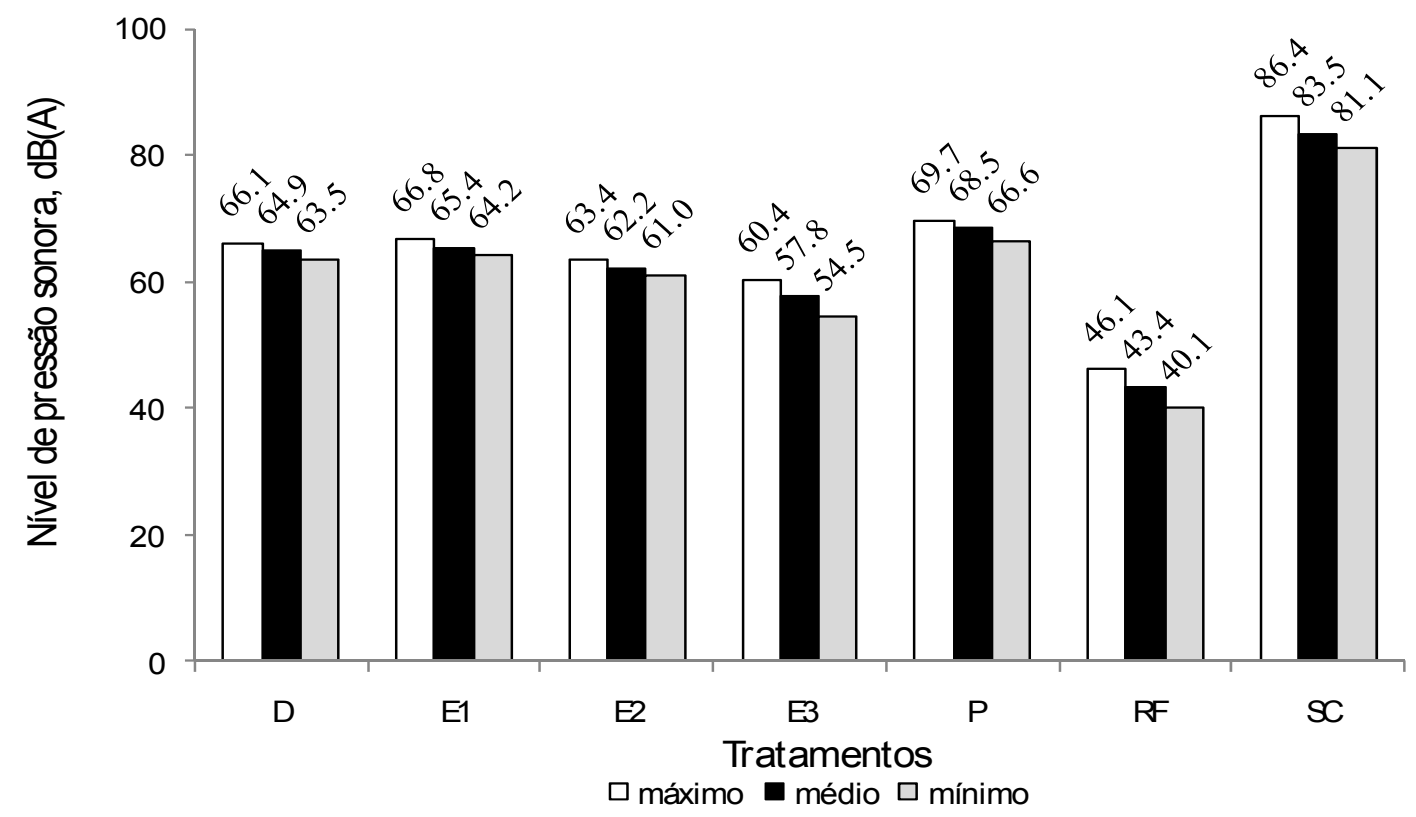

Figura 4. Valores máximos, médios e mínimos de pressão sonora obtidos nos diferentes tratamentos. D: Dipteryx sp. $\left(0,817 \mathrm{~g} / \mathrm{cm}^{3}\right)$; E1: E. grandis $\left(0,728 \mathrm{~g} / \mathrm{cm}^{3}\right)$; E2: E. grandis $\left(0,799 \mathrm{~g} / \mathrm{cm}^{3}\right)$; E3: E. grandis $\left(0,880 \mathrm{~g} / \mathrm{cm}^{3}\right)$; P: Pinus sp. $\left(0,445 \mathrm{~g} / \mathrm{cm}^{3}\right)$; RF: ruído de fundo; SC: sem barreira acústica.

Figure 4. Maximum, mean and minimum values of sound pressure obtained in the different woods. D: Dipteryx sp. $\left(0,817 \mathrm{~g} / \mathrm{cm}^{3}\right)$; E1: E. grandis $(0,728 \mathrm{~g} / \mathrm{cm} 3)$; E2: E. grandis $(0,799 \mathrm{~g} / \mathrm{cm} 3)$; E3: E. grandis $(0,880 \mathrm{~g} / \mathrm{cm} 3)$; P: Pinus sp. $(0,445 \mathrm{~g} / \mathrm{cm} 3)$; RF: background noise: SC: characterization of sound source.

Na tabela 7 encontra-se o resumo da análise de variância dos níveis de pressão sonora. Observase que houve diferença significativa, a 5\% de significância, para os tratamentos utilizados.

Tabela 7. Análise de variância para o nível de pressão sonora.

Table 7. Variance analysis for sound pressure level.

\begin{tabular}{lccc}
\hline Fonte de variação & Grau de liberdade & Quadrado médio & Fc \\
\hline Tratamento & 6 & 13053,50 & $15380,123^{*}$ \\
Erro & 623 & 0,848726 & \\
\hline Total & 629 & & \\
\hline
\end{tabular}

Na tabela 8 encontra-se a comparação múltipla entre as médias do nível de pressão sonora. Podese afirmar que, estatisticamente, todas as madeiras apresentaram níveis de pressão sonora diferentes. A madeira que apresentou menor poder de isolamento foi o Pinus sp, justamente a que apresentou também a menor densidade. De modo geral, essa situação foi predominante, ou seja, quanto menor a densidade da espécie, menor também o isolamento proporcionado, confirmando a Lei da Massa (SILVA, 2005). 
Tabela 8. Comparação múltipla entre as médias do nível de pressão sonora, em $\mathrm{dB}(\mathrm{A})$, em função da espécie.

Table 8. Multiple comparison of the means of the level of sound pressure in $\mathrm{db}(\mathrm{A})$ as related to the species.

\begin{tabular}{lcc}
\hline Tratamento & Densidade $\mathbf{( g / \mathbf { c m } ^ { 3 } )}$ & Médias \\
\hline Sem caixa & & $83,5 \mathrm{a}$ \\
Pinus sp. & 0,445 & $68,5 \mathrm{~b}$ \\
E. grandis - 1 & 0,728 & $65,4 \mathrm{c}$ \\
Dipteryx sp. (cumaru) & 0,817 & $64,9 \mathrm{~d}$ \\
E. grandis - 2 & 0,799 & $62,2 \mathrm{e}$ \\
E. grandis - 3 & 0,880 & $57,8 \mathrm{f}$ \\
Ruído de fundo & & $43,4 \mathrm{~g}$ \\
\hline Médias seguidas pela mesma letra não diferem entre si, a 5\% de significância, pelo teste SNK.
\end{tabular}

\section{CONCLUSÕES}

- A caracterização da fonte sonora apresentou uma distorção quanto ao ruído aleatório gerado pelo computador.

- Ocorreu uma redução do nível de pressão sonora ao se utilizarem as barreiras acústicas com madeiras de diferentes densidades como anteparos.

- O tratamento que apresentou maior isolamento foi o E. grandis $\left(0,880 \mathrm{~g} / \mathrm{cm}^{3}\right)$, com uma redução de 25,7 dB(A). O menor isolamento foi apresentado pelo Pinus sp., com uma atenuação de $15 \mathrm{~dB}(\mathrm{~A})$.

- Houve uma queda no isolamento ao se utilizarem madeiras com menor densidade aparente.

\section{REFERÊNCIAS}

ARAÚJO, H. J. B. Agrupamento das espécies madeireiras ocorrentes em pequenas áreas sob manejo florestal do projeto de colonização Pedro Peixoto (AC) por similaridade das propriedades físicas e mecânicas. 157 p. Dissertação (Mestrado em Recursos Florestais) - Universidade de São Paulo, Piracicaba, 2002.

ASSOCIAÇÃO BRASILEIRA DE NORMAS TÉCNICAS (ABNT). NBR12179 - Tratamento acústico em recintos fechados. [São Paulo], 1992. 9 p.

ASSOCIAÇÃO BRASILEIRA DE NORMAS TÉCNICAS (ABNT). NBR10151 - Avaliação do ruído em áreas habitadas, visando o conforto da comunidade - Procedimento. São Paulo, 2000. 4 p.

ASSOCIAÇÃO BRASILEIRA DE NORMAS TÉCNICAS (ABNT). NBR11941 - Madeira Determinação da densidade básica. São Paulo, 2003. 6 p.

ASSOCIAÇÃO BRASILEIRA DE NORMAS TÉCNICAS (ABNT). NBR7190 - Projetos em estruturas de madeira. São Paulo, 1997. 107 p.

BISTAFA, S. R. Acústica aplicada ao controle do ruído. São Paulo: E. Blüncher, 2006. 368 p.

CAliXto, W. P.; RODRIGUES, C. G. Poluição sonora. Goiânia: Universidade Católica de Goiás. 2004. $105 \mathrm{p}$.

CARRASCO, E. V. M.; AZEVEDO JÚNIOR, A. P. Avaliação não destrutiva de propriedades mecânicas de madeiras através de ultra-som - fundamentos físicos e resultados experimentais. CERNE, Lavras, v. 9, n. 2, p. 178-191, jul./dez. 2003.

CLÍMACO, R. S. C. Conforto Sonoro: notas de aula. Brasília, DF: Faculdade de Arquitetura e Urbanismo, UnB, 2003. 47 p.

COMPANHIA DE TECNOLOGIA DE SANEAMENTO AMBIENTAL (CETESB). Norma Técnica L11.031 - Ruído - Determinação do nível de ruído de fundo - Método de ensaio. São Paulo, 1986. 
CRUZ, C. R. Aplicação de ondas de tensão para a estimativa da umidade em madeira de Eucalyptus. 72 p. Tese (Doutorado em Engenharia Florestal) - Setor de Ciências Agrárias, Universidade Federal do Paraná, Curitiba, 2006.

FAGUNDES, P. V. Utilização de espécies madeireiras amazônicas para fabricação do corpo da gaita diatônica. 55 p. Trabalho final de curso (Engenharia Florestal) - Universidade de Brasília, Brasília, DF, 2003.

FERNANDES, G. A.; Avaliação de madeiras brasileiras para utilização em guitarras elétricas.. 41 p. Trabalho final de curso (Engenharia Florestal) - Universidade de Brasília, Brasília, DF, 2004

FERNANDES, J. C. Acústica e Ruídos. Bauru: UNESP, 2002. Apostila.

HERRERA, C. G.; GUIMARÃES, G. P.; YEHIA, H. C.; MEDEIROS, E. B. Método para Avaliação Acústica de Salas Baseado em TSP. In: SEMEA 2002. SEMINÁRIO DE ENGENHARIA DE ÁUDIO, 1., 2002, Belo Horizonte. Anais... Belo Horizonte: Departamento de Engenharia Eletrônica / UFMG, 2002.

OITICICA, M. L. G. R.; GOMES, M. L. B. O estresse do professor acentuado pela precariedade das condições acústicas das salas de aula. In: ENCONTRO NACIONAL DE ENGENHARIA DE PRODUÇÃO, 14., 2004, Florianópolis. Anais... Rio de Janeiro: ABREPO, 2004, p. 2539-2546.

OKUNO, E.; CALDAS, C. L.; CHOW, C. Física para ciências biológicas e biomédicas. São Paulo: Harbra, 1986. 490 p.

PAIXÃO, D. X. Caracterização do isolamento acústico de uma parede de alvenaria, utilizando a análise estatística de energia (SEA). Tese (Doutorado em Engenharia de Produção) - Universidade Federal de Santa Catarina, Florianópolis, 2002.

PIMENTEL-SOUZA, F. Efeito do ruído no homem dormindo e acordado. In: ENCONTRO DA SOCIEDADE BRASILEIRA DE ACÚSTICA, 19., 2000, Belo Horizonte. Anais... Belo Horizonte, 2000. p. $90-108$.

PUCCINI, C. T. Avaliação de aspectos de qualidade da madeira utilizando o ultra-som. Tese (Doutorado em Engenharia Agrícola) - Universidade Estadual de Campinas, Campinas, 2002.

SEEP, B.; GLOSEMEYER, R.; HULCE, E.; LINN, M.; AYTAR, P. Acústica de salas de aula. Acústica \& Vibrações, Florianópolis, n. 29, p. 22, jul. 2002.

SHIMOYAMA, V. R. S. Estimativas de propriedades da madeira de Pinus taeda através do método não destrutivo emissão de ondas de tensão, visando a geração de produtos de alto valor agregado. Tese (Doutorado em Engenharia Florestal) - Setor de Ciências Agrárias, Universidade Federal do Paraná, Curitiba, 2005.

SILVA, P. Acústica arquitetônica e condicionamento de ar. 5. ed. Belo Horizonte: EDTAL E.T. LTDA, 2005. 339 p.

SOUZA, M. R. Classificação de madeiras para instrumentos musicais. Série técnica, Brasília, DF, n. 6 , 1983.

STEWART, H. A.; POLAK, D. J. Relating specifc gravity and mechanical properties of hardwoods to maching defects. Forest Products Journal, Medison, v. 35, n. 10, p. 69-72. 1975.

TARGA, L. A.; BALlARIN, A. W.; BIAGGIONI, M. A. M. Avaliação do módulo de elasticidade da madeira com uso de método não-destrutivo de vibração transversal. Engenharia Agrícola, Jaboticabal, v. 25, n. 2, p. 291-299, mai./ago. 2005.

TELES, R. F. Avaliação de madeiras amazônicas para utilização em instrumentos musicais - Madeiras para violões. Relatório final de projeto PIBIC. Brasília: UnB. 2004. 28 p. 